\title{
Permanent bedforms in a theoretical model of wave-sea-bed interactions
}

\author{
J. M. Becker and D. Bercovici \\ Department of Geology and Geophysics, University of Hawaii at Manoa. Honolulu. HI. 96822. USA
}

Received: 16 December 1999 - Accepted: 16 March 2000

\begin{abstract}
The interaction between sea waves and a deformable sea-bed is studied with a simple two-layer model in which the upper-layer fluid is inviscid and the lower-layer fluid is bi-viscous to account for nonNewtonian behavior of sand and sediments. The nonlinear response of the system to periodic forcing by an external surface pressure is determined. It is shown that a simple bi-viscous rheology allows small wavelength morphology in the lower layer to be generated from large wavelength surface waves in the upper inviscid layer, although the morphology is not permanent. For a biviscous rheology with a pressure-dependent yield stress (which accounts for the fact that sand yields less readily under loading than unloading), however, smail wavelength and permanent features are formed in the seabed.
\end{abstract}

\section{Introduction}

Understanding the morphological response of the nearshore to ocean waves is a long-standing coastal problem. The interaction of flow that is often turbulent with a granular medium leads to a highly complex system and no consensus has yet been reached about how best to model the resulting dynamics (Schoonees and Theron, 1995; Komar, 1998). Still, observations reveal that beaches often exhibit regular morphologic features such as sand ripples and longshore bars and troughs and a variety of modeling efforts have been conducted to gain insight into the formation of these features (c.f Blondeaux, 1990; Vittori and Blondeaux, 1990; Carter et al., 1973; Lau and Travis, 1973; Boczar-Karakiewicz and Davidson-Arnott, 1987).

Here, we examine fundamental processes leading to pattern formation in the nearshore by considering a simplified, nonlinear model. As pointed out by Holman

Correspondence to: Janet M. Becker
(1995), a promising way to gain new insight into the evolution of beach morphology is to consider simplified models that capture the essential nonlinear dynamics of the coupled ocean-sea-bed system. This follows from the hypothesis that the details of the turbulent wavy boundary layer are less important than the dynamics that govern the coupled wave-seabed system in the formation of some types of beach morphologies. We note that in many models of beach morphology, the masstransport velocity due to the wave forcing provides a template for the underlying sea bed (c.f. Carter et al., 1973; Holman and Bowen, 1982). Nonlinear, coupled models can accomodate beach morphologies with scales that differ significantly from the wave forcing.

Our dynamical modeling effort is based upon the hypothesis that sand may be modeled as a continuum with a non-Newtonian rheology. With a non-Newtonian rheology, it is possible to approximate critical bed shear stress, the most important feature of sand motion (i.e. the sand bed will deform given a sufficiently large stress, but will retain a solid structure otherwise). Recently, non-Newtonian rheologies have been used to model mud flows (Liu and Mei, 1989; Ng and Mei, 1994), the interaction of sea waves with a muddy sea bed (Liu and Mei, 1993a,b) and underwater landslides that generate tsunamis (Jiang and LeBlond, 1993).

Studies of the interaction of sea waves with a muddy seabed are most closely related to the present work. Liu and Mei (1993a,b) consider the interaction of long waves with an underlying muddy bed, modeled as a Bingham plastic. They determine the coupled response of a two-layer system to forcing by a localized initial freesurface displacement. The transient waves that radiate from this initial disturbance interact with and leave be hind permanent imprints in the Bingham plastic layer. Their study, however, does not address whether persistent forcing by periodic surface waves would excite regular features in the mud that maintain their structure over many periods of the forcing. 
2

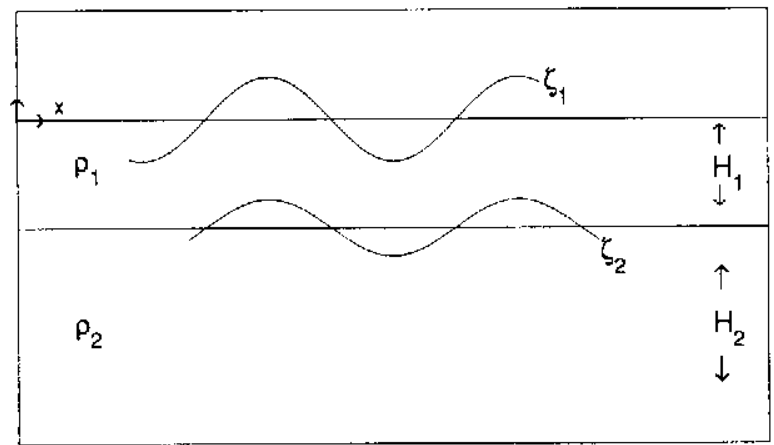

Fig. 1. The two layer model. Both the upper and lower layer fluids are incompressible with constant densities $\rho_{1}$ and $\rho_{2}$. The upper layer is inviscid while the lower layer fluid is bi-viscous (see (5)).

Thus, our study differs from that of Liu and Mei $(1993 a, b)$ in two important ways. First, we consider the model response to periodic forcing rather than an initial localized disturbance to determine how the length and time scales of the lower-layer response are related to the length and time scales of the forcing. Second, as we are interested in the interaction of sea-waves with sand rather than mud, we consider different rheologies for the deformable lower layer in an effort to obtain a better rheological model for sand. In particular, the simple Bingham rheology is not always appropriate for sand; for example, for flow down an incline, motion will initiate from the top for sand, but from the bottom for a Bingham plastic (Jaeger and Nagel, 1992). Such behavior suggests that for sand motion, the yield stress is pressure dependent (i.e. sand yields less readily when under a load).

We start with a bi-viscous rheology (the Bingham rheology is a limiting form of the bi-viscous rheology as the first viscosity becomes infinite) for the lower layer. We choose this rheology because it represents simply the two flow states of sand: sand resists motion for small applied stresses but flows readily for stresses above a critical value. We find that the nonlinearity inherent in this rheology generates small-scale spatial features in the lower layer, but that these spatial features oscillate with the forcing frequency. As a result, permanent features are not excited in a bi-viscous lower layer. By allowing for a pressure-dependent yield stress so that the rheology depends upon the history of the loading, however, we find that permanent small-scale spatial features are excited in the deformable lower layer.

\section{Dynamics}

We consider here two superposed shallow homogeneous layers of fluid (Fig. 1).

The upper layer fluid is inviscid and incompressible with constant density $\rho_{1}$ and is bounded above by a free surface located at $z=\zeta_{1}(x, t)$ and below by an interface located at $z=\zeta_{2}(x, t)-H_{1}$ where $H_{1}$ is the equilibrium depth of the upper layer. The lower layer fluid is non-Newtonian and incompressible with constant density $\rho_{2}$ and is bounded above by the interface at $z=\zeta_{2}(x, t)-H_{1}$ and below by a rigid bottom located at $z=-\left(H_{1}+H_{2}\right)$ where $H_{2}$ is the equilibrium depth of the lower layer. To drive the wave motion, we impose an external pressure $P_{0}(x, t)=A \cos (k x) \cos (\omega t)$ of amplitude $A$, wavenumber $k$ and frequency $\omega$ on the free surface.

The forced long-wave equations in the absence of inertia governing this two-layer system are

$$
\begin{aligned}
& \frac{\partial u_{1}}{\partial t}=-g \frac{\partial \zeta_{1}}{\partial x}-\frac{1}{\rho_{1}} \frac{\partial P_{0}}{\partial x} \\
& \frac{\partial u_{2}}{\partial t}=-g \frac{\partial \zeta_{2}}{\partial x}-\frac{\rho_{1}}{\rho_{2}} g \frac{\partial\left(\zeta_{1}-\zeta_{2}\right)}{\partial x}-\frac{\tau_{b}}{\rho_{2} H_{2}}-\frac{1}{\rho_{2}} \frac{\partial P_{0}}{\partial x} \\
& \frac{\partial\left(\zeta_{1}-\zeta_{2}\right)}{\partial t}+H_{1} \frac{\partial u_{1}}{\partial x}=0 \\
& \frac{\partial \zeta_{2}}{\partial t}+H_{2} \frac{\partial u_{2}}{\partial x}=0
\end{aligned}
$$

where $u_{j}(x, t)(j=1,2)$ is the horizontal upper/lower layer velocity, $g$ is the gravitational acceleration and $\tau_{b}$ is the bottom stress. We remark that inertia is neglected in (1)-(4) to focus on the effects of the nonlinear rheology (see (5) below).

To close this system, we specify the rheology of the lower layer. As mentioned in the introduction, a simple model of the two flow states of sand is a bi-viscous rheology for which the bottom stress $\tau_{b}$ is related to the strain rate, here approximated as $\frac{u_{2}}{H_{2}}$ according to:

$$
\begin{aligned}
& \frac{1}{\mu} \tau_{b}=\frac{u_{2}}{H_{2}}, \tau_{b} \leq \tau_{0} \\
& \frac{1}{\mu^{\prime}} \tau_{b}=\tau_{0}\left(\frac{1}{\mu^{\prime}}-\frac{1}{\mu}\right) \operatorname{sgn}\left(\frac{u_{2}}{H_{2}}\right)+\frac{u_{2}}{H_{2}}, \tau_{b}>\tau_{0}
\end{aligned}
$$

In (5), $\tau_{0}$ is the yield stress, and $\mu$ and $\mu^{\prime}$ are the dynamic viscosities. We remark that in the limit $\mu \rightarrow \mu^{\prime}$, (5) describes a Newtonian fluid, whereas in the limit $\mu \rightarrow \infty,(5)$ describes a Bingham plastic.

We next nondimensionalize (1)-(5) according to

$$
\begin{aligned}
(x, t) & \rightarrow L\left(x, c^{-1} t\right) \\
\left(u_{j}, \zeta_{j}\right) & \rightarrow \frac{A k L}{\rho_{1} c^{2}}\left(c u_{j}, H \zeta_{j}\right) \quad(j=1,2) \\
\left(P_{0}, \tau_{b}\right) & \rightarrow\left(A P_{0}, A k H \frac{\rho_{2}}{\rho_{1}} \tau_{b}\right)
\end{aligned}
$$

where $L$ is a representative horizontal lengthscale, $H=$ $H_{1}+H_{2}$ and $c=\sqrt{g H}$ is the long wave speed. In addition, for spatially periodic forcing, we choose the nondimensional wavenumber of the forcing as $k L=m \pi$ where $m$ is an integer.

Then, the dimensionless governing equations become 
$\frac{\partial u_{1}}{\partial t}=-\frac{\partial \zeta_{1}}{\partial x}+\sin (m \pi x) \cos (\Omega t)$

$\frac{\partial u_{2}}{\partial t}=-\frac{\partial \zeta_{2}}{\partial x}-s \frac{\left(\partial \zeta_{1}-\zeta_{2}\right)}{\partial x}$ $-\frac{\tau_{b}}{H_{2}^{*}}+s \sin (m \pi x) \cos (\Omega t)$

$\frac{\partial\left(\zeta_{1}-\zeta_{2}\right)}{\partial t}+H_{1}^{*} \frac{\partial u_{1}}{\partial x}=0$

and

$\frac{\partial \zeta_{2}}{\partial t}+H_{2}^{*} \frac{\partial u_{2}}{\partial x}=0$

where

$\frac{1}{\Gamma} \tau_{b}=\frac{u_{2}}{H_{2}^{*}}, \quad \tau_{b} \leq \Upsilon_{0}$

$\frac{1}{\Gamma^{\prime}} \tau_{b}=\Upsilon_{0}\left(\frac{1}{\Gamma^{\prime}}-\frac{1}{\Gamma}\right) \operatorname{sgn}\left(\frac{u_{2}}{H_{2}^{*}}\right)+\frac{u_{2}}{H_{2}^{*}}, \quad \tau_{b}>\Upsilon_{0}$

In (6)-(10), the controlling parameters include the density and layer depth ratios: $s=\frac{\rho_{1}}{\rho_{2}}$ and $H_{j}^{*}=\frac{H_{j}}{H_{1}+H_{2}}(j=$ $1,2)$, and the nondimensional viscosity parameters, yield stress and forcing frequency: $\left(\Gamma, \Gamma^{\prime}\right)=\frac{L}{c \rho_{2} H^{2}}\left(\mu, \mu^{\prime}\right)$, $\Upsilon_{0}=A k H \frac{\rho_{2}}{\rho_{1}} \tau_{0}$ and $\Omega=\frac{\omega c}{L}$. We note that $H_{1}^{*}+H_{2}^{*}=1$.

\section{Numerical Solutions}

We solve (6)-(10) starting from rest and subject to periodic boundary conditions on $0<x<2$. We use a pseudospectral method and de-alias the nonlinear terms following Canuto et al. (1988), pages 83-85. We determine the time-dependent Fourier amplitudes by replacing time derivatives in (6)-(10) by a centered-difference scheme and time-stepping the resulting equations in wavenumber space until a steady state is achieved.

In the numerical solutions that follow, we use a spatial resolution of 128 grid points which is sufficient for all cases examined. In addition, we fix the density and layer depth ratios and the first viscosity parameter as: $s=\frac{1}{1.1}, H_{1}^{*}=\frac{1}{3}, \Gamma=50$, and fix the forcing wavenumber and frequency according to $\kappa=m \pi=3 \pi$ and $\Omega=3 \pi \sqrt{H_{1}^{*}}$ so that the forcing frequency obeys the dispersion relationship for standing long waves on a layer of fluid of depth $H_{1}^{*}$ (i.e. the lower layer appears rigid to the upper layer fluid in this approximation).

\subsection{Newtonian Rheology}

We first consider the solution of (6)-(10) in the Newtonian limit for which $\Gamma^{\prime} \rightarrow \Gamma=50$. In the Newtonian limit, the dynamics are linear and it is straight-forward to write down an analytic solution in this approximation. Our aim, however, is to compare this solution with those for which the lower layer rheology is nonlinear; hence, we present this solution in a form to facilitate this comparison.
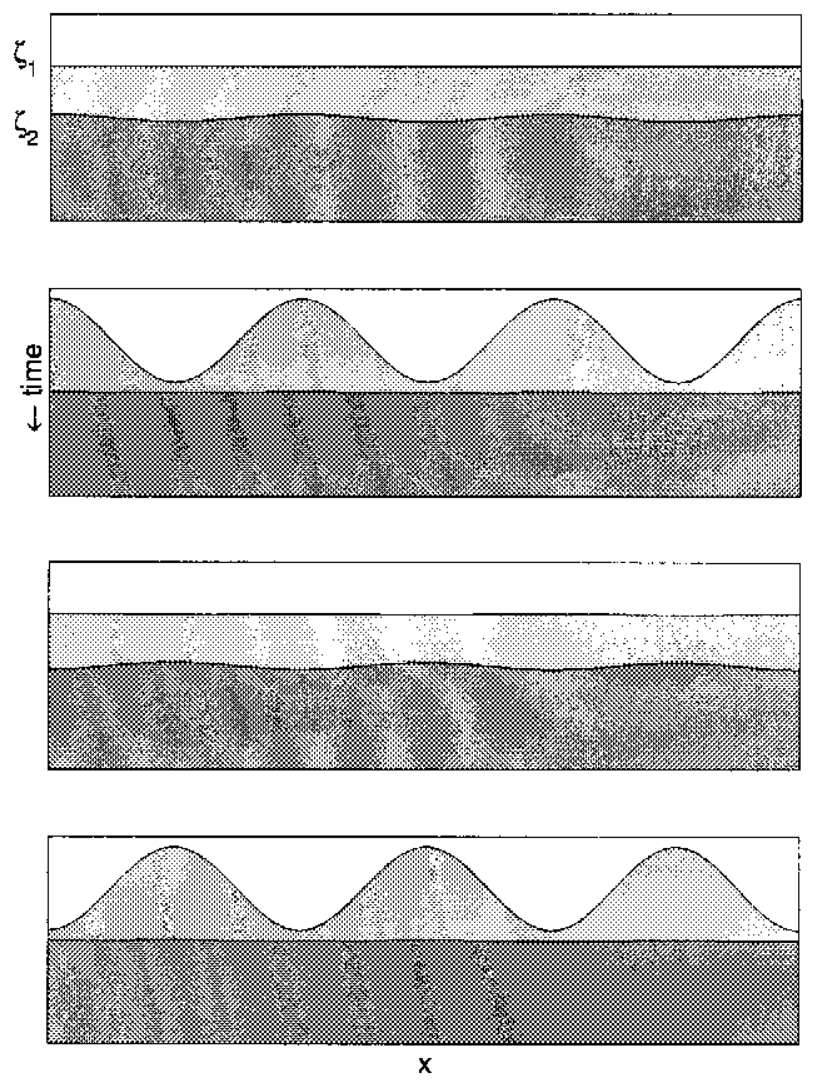

Fig. 2. Newtonian lower layer. Snapshots of the interface displacement and the free-surface displacement at four times separated by approximately $\Delta t=\frac{\pi}{2 \Omega}=0.29$. The time increases from top to bottom. The controlling parameters for this solution are $\left(s, H_{1}^{*}, \Gamma, \Gamma^{\prime}\right)=\left(\frac{1}{1.1}, \frac{1}{3}, 50,50\right)$ and $(m, \Omega)=(3, \sqrt{3} \pi)$.

In the Newtonian limit, only the directly-forced mode with a wavelength of $\lambda_{0}=\frac{2}{3}$ (with nondimensional wavenumber $\kappa=3 \pi$ ) is excited. Fig. 2 presents snapshots of the interface and free-surface displacements at four times separated by approximately one-fourth of the period of the forcing $\left(\Delta t=\frac{\pi}{2 \Omega}\right)$. The dissipation in the lower layer has caused a temporal phase shift between the free-surface and interface displacements and the relationship between the amplitudes of the free-surface and interface displacements is consistent with the excitation of a damped internal wave mode.

\subsection{Bi-viscous rheology}

We next consider the solution of (6)-(10) but with a bi-viscous rheology for the lower layer (i.e. $\Gamma \neq \Gamma^{\prime}$ ). Fixing the second viscosity and yield stress as $\Gamma^{\prime}=1$ and $\Upsilon_{0}=1.5$, numerical solutions reveal that energy is exchanged among the directly-forced mode with $\lambda_{0}=\frac{2}{3}$ and modes with smaller wavelengths that are related to this fundamental wavelength by odd integers:

$\lambda_{n}=\frac{\lambda_{0}}{n} \quad(n=3,5,7 \ldots)$ 

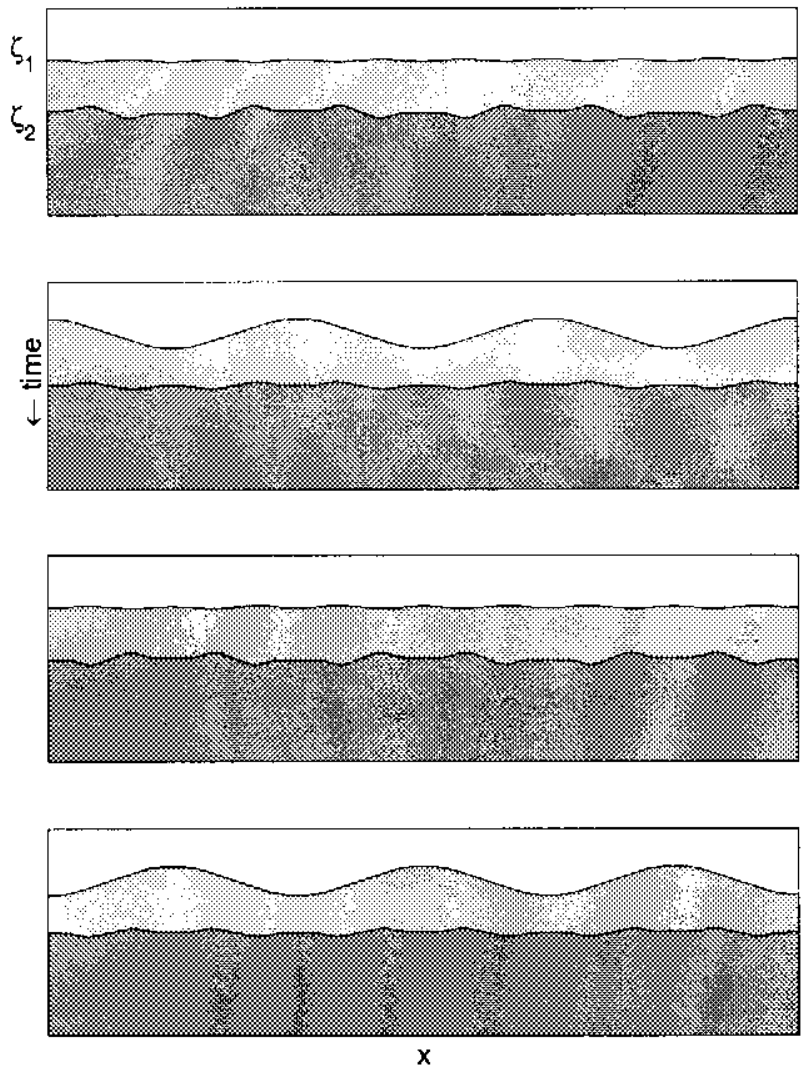

Fig. 3. Bi-viscous lower layer. Same as Figure 2, but for the controlling parameters $\left(s, H_{1}^{*}, \Gamma, \Gamma^{\prime}, \Upsilon_{0}\right)=\left(\frac{1}{1.1}, \frac{1}{3}, 50,1,1.5\right)$ and $(m, \Omega)=(3, \sqrt{3} \pi)$.

We emphasize that in this simple model, the only nonlinearity in this solution is due to the bi-viscous rheology.

Fig. 3 presents snapshots of the free-surface and interface displacements at four times analgous to those in Fig. 2. The significant energy transfer to the smaller wavelength modes is evident in the interface displacements. We remark that there is more energy in the $\lambda_{3}=\frac{2}{9}$ mode than in the $\lambda_{0}=\frac{2}{3}$ mode and that the $\lambda_{5}=\frac{2}{15}$ mode also has significant energy.

Fig. 3 also shows that both the interface and freesurface displacements oscillate with the forcing frequency. The time-average of these displacements over a period of the forcing is zero; hence, permanent features in the lower layer are not established for the bi-viscous rheology. We also remark that solutions of (6)-(10) for a larger viscosity contrast (e.g. $\Gamma=500, \quad \Gamma^{\prime}=1$ ) that more closely approximates the Bingham-plastic limit also reveal spatial patterns with wavelengths related by (11) that oscillate with the forcing frequency.

\section{$3.3 \quad$ Pressure-dependent yield stress}

We next consider a lower-layer rheology that models the observation that sand is more difficult to move under loading than unloading. To this end, we modify the rheology in (5) to accomodate a pressure-dependent yield
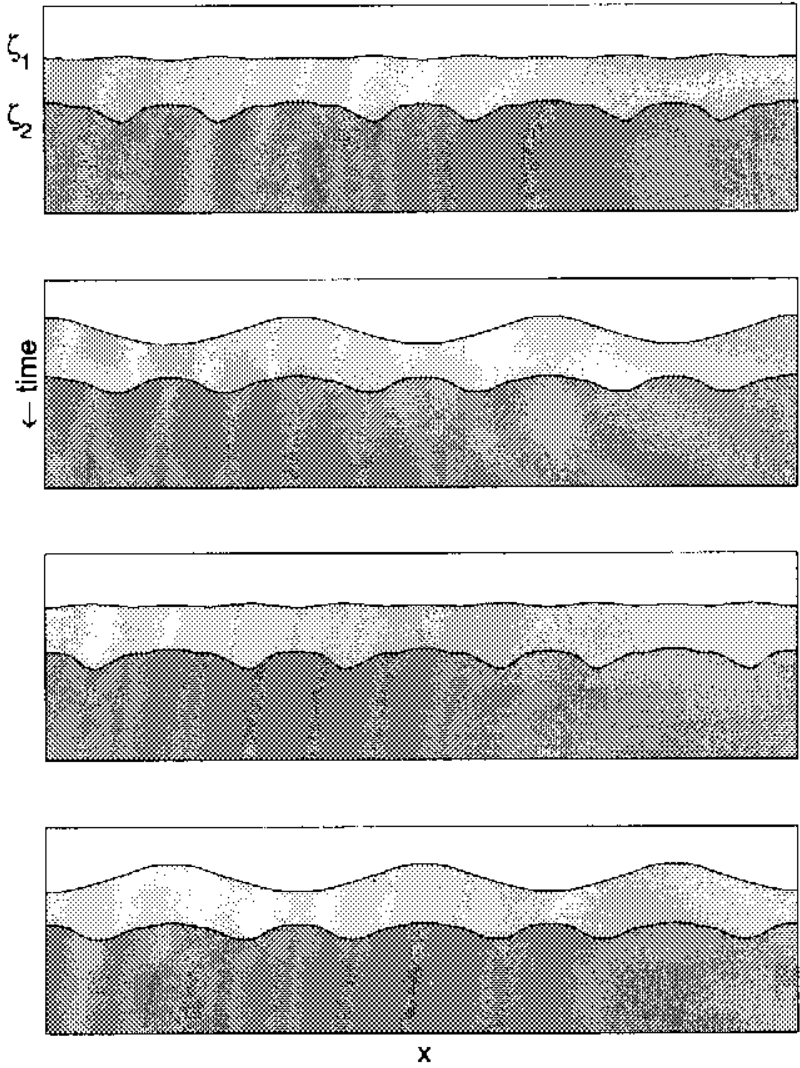

Fig. 4. Bi-viscous lower layer with a pressure-dependent yield stress. Same as Figure 3, but with $\Upsilon_{0}$ replaced by $\Upsilon_{0 p}(x, t),(12)$ with $C=2.2$.

stress given by

$\Upsilon_{0 p}(x, t)=\Upsilon_{0}\left(1+C p_{b}(x, t)\right)$

where $C$ is a constant and $p_{b}(x, t)=s\left[P_{0}(x, t)+\zeta_{1}(x, t)-\right.$ $\left.\zeta_{2}(x, t)\right]+\zeta_{2}(x, t)$ is the dimensionless dynamic bottom pressure. We then solve (6)-(10) with $\Upsilon_{0}$ replaced by $\Upsilon_{0 p}(x, t)$ therein. We choose $C$ empirically so that the pressure-dependent yield stress, $\Upsilon_{0 p}$ undergoes significant variations, but always remains positive. The runs presented have $C=2.2$ for which the pressure-dependent yield stress varies from 0.4 to 2.9 .

Here, numerical solutions reveal that energy is exchanged among the directly-forced mode and those mode: that are related to this fundamental wavelength by odd and even integers: $\lambda_{n p}=\frac{\lambda_{0}}{n}(n=2,3,4 \ldots)$. Again, the only nonlinearity in this simple model is due to the rheology, but here the rheology depends upon the history of the loading.

Fig. 4 presents the four snapshots of the interface and free-surface displacements at times that correspond to those in Figs. 2 and 3. In this case with the pressuredependent yield stress, the mode with wavelength $\lambda_{2 p}=$ $\frac{1}{3}$ has the most energy. We emphasize that this mode was not excited in the constant yield stress case. Similar to the constant yield stress case, the $\lambda_{3 p}=\frac{2}{9}$ mode, the 
directly-forced $\lambda_{0}=\frac{2}{3}$ mode and the $\lambda_{5 p}=\frac{2}{15}$ mode also have significant energy.

Moreover, the plots of the interface displacement in Fig. 4 show that the spatial pattern formed in the lowerlayer has a significant non-zero time average over a period of the forcing. The pressure-dependent yield stress has caused a permanent spatial pattern to form in the lower layer. This may be interpreted as follows: once an elevation is formed in the lower layer, the bottom pressure below this elevation increases which increases the yield stress. As a result of the higher yield stress, the fluid in the elevation is less likely to experience an applied stress that exceeds the critical stress, and hence is less likely to erode under the periodic forcing. Similarly, under a depression in the lower layer, the bottom pressure and yield stress decrease, and the fluid in a depression is easier to erode.

\section{Discussion}

We have presented here a simple dynamical model of the coupled motion of long waves over a deformable sea bed in an initial effort to model pattern formation in the nearshore. This model is based upon the hypothesis that sand may be modeled as a continuum with a nonNewtonian rheology.

Using a bi-viscous rheology that is perhaps the simplest continuum model of the two flow states of sand, we have demonstrated that energy may be transferred to wavelengths that are smaller than the wavelength of the forcing. That these wavelengths are related to the wavelength of the forcing by odd integers (see (11)) is consistent with the bottom stress being an odd function of the strain rate. The spatial pattern formed in the deformable lower layer, however, oscillates with the forcing frequency so permanent features in the lower layer do not form with the bi-viscous rheology.

By considering a more realistic (although still highly idealized) rheology for the deformable lower layer, for which we model the effect that sand is more difficult to move under loading than unloading, we find that additional wavelengths are excited in the lower layer. Moreover, the spatial pattern in the deformable lower layer has a significant non-zero time average which demonstrates that permanent features may form in the lower layer with a pressure-dependent yield stress.

Finally, we remark that for the pressure-dependent yield stress, the dominant wavelength of the permanent lower-layer feature is half that of the forcing. This result is consistent with laboratory experiments on the formation of sand bars in a wave flume (e.g. O'Hare and Davies, 1990) and the previous theoretical study of sand bar formation by reflected surface waves (Carter et al., 1973). The present theory, however, differs from that of Carter et al. (1973) as it does not rely on the mass transport velocity in the Stokes boundary layer in the formation of the morphology, and does include the coupling between the evolving lower-layer morphology and the wave forcing.

Acknowledgements. This paper is funded in part by a grant/ cooperative agreement from the National Oceanic and Atmospheric Administration, project \# R/EP-9, which is sponsored by the University of Hawaii Sea Grant College Program, SOEST, under Institutional Grant No. NA86RG0041 from NOAA office of Sea Grant, Department of Commerce. The views expressed herein are those of the authors and do not necessarily reflect the views of NOAA of any of its subagencies. UNIHI- SEAGRANT-JC-99-14. $\mathrm{JB}$ also is supported by by NSF-OCE-9633057. This is SOEST contribution no. 5196 .

\section{References}

Blondeaux, P., Sand ripples under sea waves Part 1. Ripple formation, J. Fluid Mech., 218, 1-17, 1990.

Boczar-Karakiewicz, B. and R.G.D. Davidson-Arnott, Nearshore bar formation by non-linear wave processes- a comparison of model results and field data, Marine Geology, 77, 287-304, 1987.

Canuto, C., Hussaini, M.Y., Quarteroni, A. and T.A. Zang, Spectral Methods in Fluid Dynamics, 567 pp., Springer-Verlag, Berlin Heidelberg, 1988.

Carter, T.G., P.L-F. Liu and C.C. Mei, Mass transport by waves and offshore sand bedforms, J. Waterways, Harbors, and Coastal Eng. Div., ASCE, 99(WW2) 165-184, 1973.

Holman, R.A., The interaction of waves, currents and nearshore bathymetry, Proceedings 'Aha Huliko'a Hawaiian Winter Workshop, edited by P. Muller and D. Henderson, 257-262, SOEST Special Publication, 1995.

Holman, R.A. and A.J. Bowen, Bars, bumps, and holes: models for the generation of complex beach topography, $J$. Geophys. Res. 87(C1), 457-468, 1982.

Jaeger, H.M. and S.R. Nagel, Physics of the granular state, Science, 255, 1523-1531, 1992.

Jiang, L. and P.H. LeBlond, Numerical modeling of an underwater Bingham plastic mudslide and the waves which it generates, $J$. Geophys. Res. 98(C6), 10,303-10,317, 1993.

Komar, P.D., Beach Processes and Sedimentation, 544pp., Prentice Hall, New Jersey, 1998.

I.au, J. and B. Travis, Slowly varying Stokes waves and submarine longshore bars, J. Geophys. Res. 78(21), 4489-4497, 1973.

Liu, K-F. and C.C. Mei, Slow spreading of a sheet of Bingham flud on an inclined plane, J. Fluid Mech., 207, 505-529, 1989.

Liu, K-F. and C.C. Mei, Long waves in shallow water over a layer of Bingham-plastic fluid-rnud-I. Physical Aspects, Int. J. Engng. Sci., 31(1), 125-144, 1993a.

Liu, K-F. and C.C. Mei, Long waves in shallow water over a laver of Bingham-plastic fiuid-mud-11. Mathematical derivation of long wave equations, Int. J. Engng. Sci., 31(1), 145-155, $1993 \mathrm{~b}$.

$\mathrm{Ng}, \mathrm{C}-\mathrm{O}$. and C.C. Mei, Roll waves on a shallow layer of mud modeled as a power-law fluid, J. Fluid Mech., 263, 151-183, 1994.

O'Ilare, T.J. and A.G. Davies, A laboratory study of sand bar evolution, J. Coastal Res., 6(3), 531-544, 1990.

Schoonees, J.S. and A.K. Theron, Evaluation of 10 cross-shore sediment transport/morphological models, Coastal Eng., 25, $1-4 \mathrm{l}, 1995$.

Vittori, G. and P. Blondeaux, Sand ripples under sea waves Part 2. Finite-amplitude development, $f$. Fluid Mech., 218, 19-39, 1990 . 